\title{
University of Turin performance in UI GreenMetric Energy and Climate Change
}

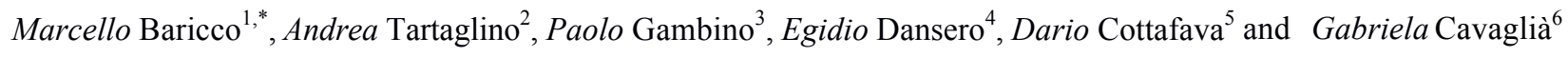 \\ ${ }^{1}$ Vice-Rector, Department of Chemistry and NIS, University of Turin, Italy \\ ${ }^{2}$ Energy Manager, University of Turin, Italy \\ ${ }^{3}$ Department of Physics, University of Turin, Italy \\ ${ }^{4}$ Sustainability Delegate, Department of Culture, Politics and Society, University of Turin, Italy \\ ${ }^{5}$ Department of Physics, University of Turin, Italy \\ ${ }^{6}$ UNESCO Chair in Sustainable Development and Territory Management, University of Turin, Italy
}

\begin{abstract}
At the University of Turin (UniTo) the first attempt to reduce the ecological footprint dates back to 2006. In 2013 UniTo participated, for the first time, to the GreenMetric World University Rankings and it launched its most ambitious sustainable initiatives by publishing the first annual University Sustainability Report. Since 2013, several efforts have been made to reduce carbon emissions, to improve the energy efficiency of buildings, as well as for reducing water consumption, improving waste management, promoting sustainable mobility, and increasing ecological purchases. The most recent achievement was in 2016, when the University Green Office (UniToGO) was established. Concerning the energy management, UniTo may be considered as a "city within a city": it counts about 70,000 students and 4,000 academic and administrative-technical staff studying and working in about 120 buildings, with an annual primary energy cost of over $10 \mathrm{M} €$. Thanks to UniToGO, UniTo adopted an Energy Plan with the aim to reduce primary energy consumption, to improve buildings energy efficiency and to increase the energy production from renewable energy. As a result, several actions relevant for GreenMetric were performed: the renovation of old chiller and substitution of new energy efficient LED, the implementation of Smart Building Systems (BEMS) for HVAC plants and the adoption of an OpenData policy for energy consumption, the increase of renewable energy production, mainly due to three cogeneration plants, and the adoption of a university policy in order to buy only renewable energy from the current Distribution System Operator. Moreover, UniTo took several efforts to improve or to design elements of green buildings in a partecipatory way. Finally, during 2017, the Environmental Sustainability Action Plan was set-up to plan future actions related to five sustainability fields: Energy, Food, Green Public Procurement, Mobility and Waste.
\end{abstract}

\section{Introduction}

Since the Rio de Janeiro Earth Summit in 1992, universities - considered as place of training of future generation - are called to assume their social responsibility and the important role of "agents of change" through targeted choices in their three spheres of intervention:

1. education, to foster the development of a culture of sustainability and the widening of the educational offer of green curricula and courses [1];

2. research, to stimulate the development of inter and multidisciplinary research projects with a holistic and innovative approach to deal with climate change and, more in general, with challenges facing $21^{\text {st }}$ century society;

3. "third mission", to develop targeted networking actions with local authorities, stakeholder engagement and active citizenship involvement [2].
The goal of reducing the university ecological footprint needs the involvement and the commitment of the entire community, from the governing bodies to students, from academia to technical- administrative staff.

Over the years, numerous universities all over the world have started a reflection on the role that the universities can play in the challenge of environmental sustainability. Many campuses have undertaken a process of improvement of their environmental performance by planning policies, strategies, projects and precise interventions. Moreover, several university networks have been developed to share experiences, ideas, solutions and best practices [3-5].

The first attempt made by the University of Turin (UniTo) to reduce its ecological footprint dates back to 2006 with the project Towards an Agenda 21 of the University, a students' initiative supported by some professors. It led to the creation of the University Environmental Sustainability Commission with the aim of proposing structural interventions on campus

\footnotetext{
*Corresponding author: marcello.baricco@unito.it
} 
sustainability [6]. Some experimental pilot projects, aimed to improve mobility and canteen management, were carried out with a participatory approach promoting the creation of Local Action Groups, open to the participation of all members of the university community. Although the initiatives were carefully monitored, the lack of dedicated human and economic resources, together with the lack of strong political will on the part of the university's top management prevented the progression of the projects, which however represented the first systematic attempt to design a university environmental policy.

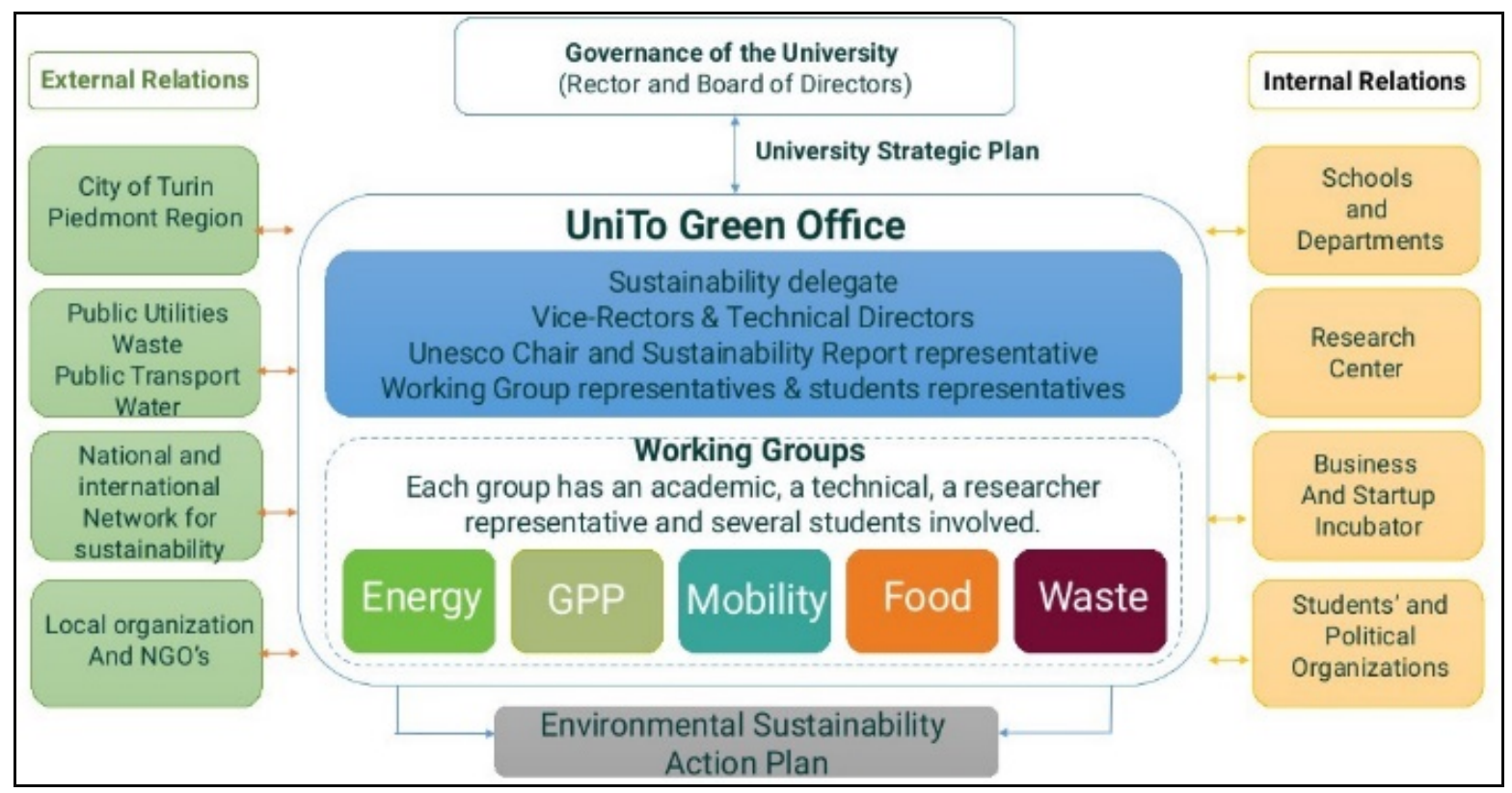

Fig. 1. Structure of the UniTo Green Office (UniToGO). The core of UniToGO is based on five working groups and a horizontal board composed by students, researchers, academic, technical and administrative staffs.

The following year, the UniTo Programming Plan for the period 2007-2012 [7] explicitly mentioned, for the very the first time, sustainable development as a reference for the UniTo's activities.

In 2009 UniTo joined the Green Public Procurement Project (APE) promoted by Turin local government [8], signing a memorandum of understanding aimed at promoting purchases with reduced environmental impact and, in 2014, the commitment to respect some minimum environmental criteria in the procedures for the purchase of goods and services. Since then, UniTo settled an internal APE working group with the aim of monitoring every year UniTo's purchases and stimulates concrete effects in terms of purchasing choices, starting from the energy field.

Since 2014 a particular effort was made for drawing up and implementing the first Energy Saving Plan [9] aimed at improving energy efficiency, reduce waste, increase the share of energy production from renewable sources and improving building efficiency and user comfort. A multidisciplinary working group,RE@UniTo, was specifically settled [10]. The activity of this group and the results achieved are described thoroughly in the second part of this paper.

The focus on environmental sustainability strengthens in the Strategic Lines of the University 2013 - 2015 by recognizing UniTo social responsibility and, even further, in the 2016 - 2020 University Strategic Plan by introducing a specific reference to the environmental dimension - "Increasing the social, economic and environmental responsibility of the University of Turin" , expressed in terms of objectives and indicators.

A fundamental step is registered with the decision to draw up and publish annually - starting from 2013/14 academic year - a Sustainability Report [11], with the purpose of analyzing and communicating publicly UniTo's performance in terms of economic, social and environmental sustainability, following the international methodology of the Global Reporting Initiative (GRI) [12]. The editing of this report is not just an assessment of UniTo's environmental impact, but also the opportunity to analyse policies and interventions carried out, to present the progress reached and to identify the steps still to be taken. The report constitutes a fundamental element in the formulation of a university environmental policy and it launched the creation of the UniTo Green Office (UniToGO).

UniToGO was created in the spring of 2016, gathering thrusts from the top (Rector and General Manager) and from the bottom (students, academia andadministrative staff), around the experience of the RE@UniTo and the APE working groups, in order to systematize UniTo knowledge and experience to promote initiatives on environmental sustainability, supported by the university community. UniToGO represents now the link between the planning addressed by UniTo governing bodies and five thematic working groups appointed to concretely develop research and actions in the various areas of intervention identified (above energy and green public procurement also, food, waste and mobility). It is a mixed and interdisciplinary 
entity made up by academic and administrative staff, together with students coming from many different disciplines within UniTo, as shown in Fig. 1. UniToGO has three main goals and lines of intervention:

- "Knowing" by building a shared knowledge base both on the initiatives launched within the UniTo and on good practices, with particular attention to the experiences of other universities in Italy and abroad;

- "Communication and Engagement" by intensifying networking actions within the university community and externally between UniTo and the relevant actors on a local, national and international scale;

- "Change" by designing and implementing interventions of various nature aimed at effectively reducing the environmental impact of the university in various areas.

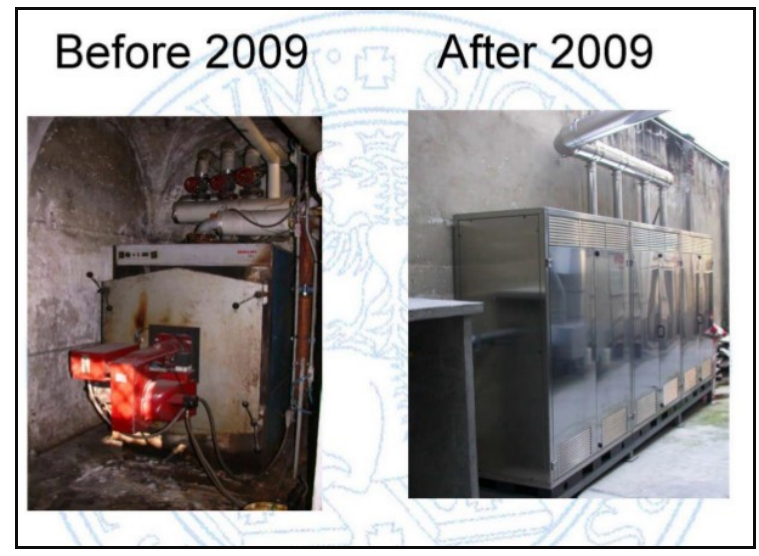

Fig. 2. Renovation of the heating plants

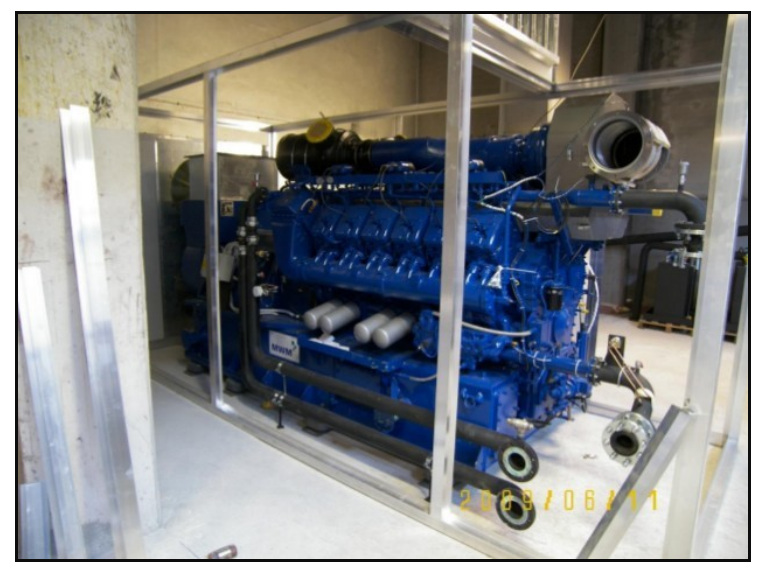

Fig. 3. Trigeneration power plant at Campus Luigi Einaudi.

\subsection{Local and international networks}

2013 was not only the year in which UniTo published its first Sustainability Report but also the first time it took part to the UI GreenMetric World University Rankings with the idea to start a comparison with other universities committed on reducing their ecological footprint. Every year, UniTo succeeded in improving its score: from a total score of 4.372 in 2013 it reached 6.064 in 2017 moving its position in the world ranking from 211 to 55 (second on the Italian rank). GreenMetric represents for UniTo not just a reliable tool for national and international comparison but also a guide to internally identify sphere of intervention.

$\mathrm{Up}$ to now, in addition to the participation to GreenMetric, UniTo extended the opportunity for comparison with other sustainable campuses network both at national and international level. UniTo joined the International Sustainable Campus Network (ISCN) [3] in 2015; it brings together over than 80 colleges and universities in more than 30 countries in a forum aimed at the exchange of information, ideas and best practices to improve environmental performance of the universities and to integrate themes related to sustainability in research and higher education.

At European level, UniTo joined the Green Office Movement [5], promoted by the Rootability association [13], which counts 17 participating universities located in 7 European countries (and 25 in the process of joining). The Green Office Movement aims to facilitate the creation of formalized working groups, made up by students supported by technical and administrative staff, to guide and coordinate the realization of ecologically sustainable and economically feasible projects. UniTo was the first Italian campus to take part in the movement, and UniToGO received the formal membership precisely for the attention dedicated to the students' involvement.

At a national level, the Network of Universities for Sustainability (RUS) was established in 2015 within the Conference of Italian University Rectors (CRUI) [14]. It counts 50 Italian universities that agreed to commit themselves to the issues of sustainability and social responsibility.

\section{Energy and climate change}

\subsection{Renovations and interventions}

The real estate assets of UniTo include around 120 locations dating back to different periods (from 18th to $21 \mathrm{st}$ centuries), for a total area of about 800,000 square meters. Since 2000 a series of restorations were carried out in the university buildings, both to renovate the facilities and to improve energy efficiency. In 2007, these efforts resulted in an important project whose total cost exceeds $€ 12$ millions. The project led to the following renovation works:

- installation of three trigenerators, with a power of $1,154 \mathrm{kWhe}$ which enable the self-production of electricity and supply heating and cooling (Fig. 2);

- transformation of all the heating plants from diesel oil to methane, with the installation of condensation boilers (Fig. 3);

- creation of a remote management system for air conditioning systems, to allow complete management of the heating and conditioning plants (Fig. 4). 


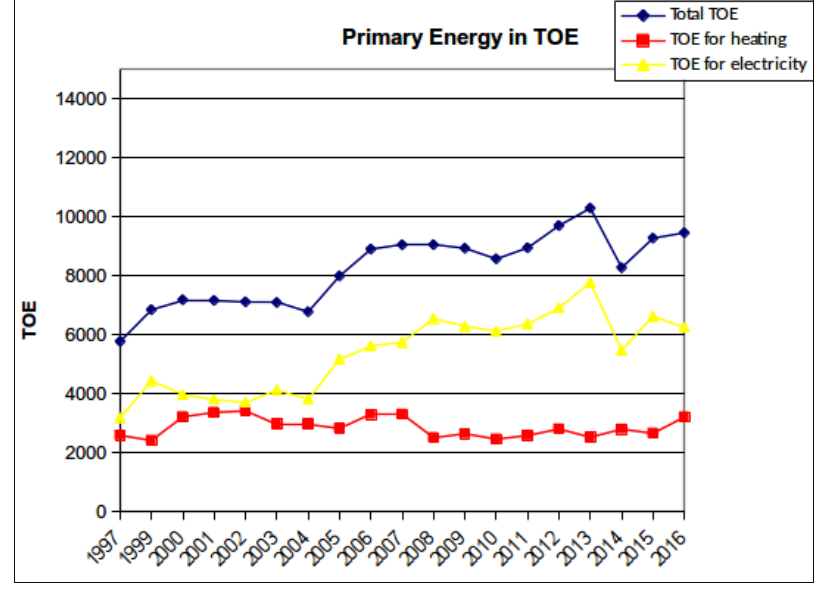

Fig. 4. Annual energy consumption trends from 1997 - 2016 of the University of Turin

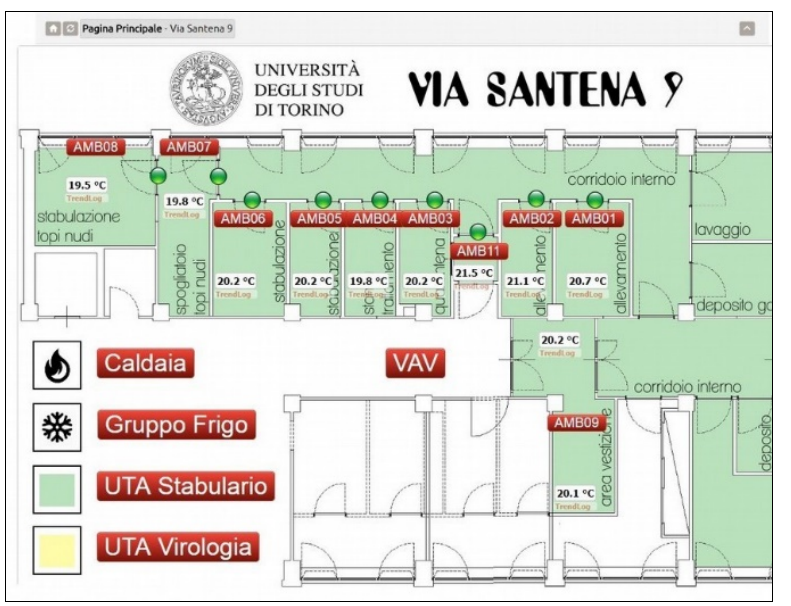

Fig. 5. A screenshot from the building energy management system

All these actions led to an annual saving of around 1.8 $\mathrm{M} €$ and 1,600 TOE, bringing the total annual cost of primary energy for UniTo to around 10.5 M€. Currently, UniTo has an annual consumption of about 10,000 TOE and the consumption trend is summarized in Fig. 5.

Thanks to the activities of RE@UniTo first and UniToGO later, in 2016 UniTo adopted an Energy Plan. This plan includes, in particular, long-term structural renovations characterized by high cost from which high long-term savings are expected, soft renovations, with limited cost and fast return, awareness-raising activities, to raise users' awareness over a conscious and rational use of energy and administrative interventions, to optimize primary energy bills. As a result, several actions relevant for GreenMetric were performed:

1. numerous renovations and energy efficiency improvements have been made with LED technology and the use of energy efficient appliances and lighting fixtures, as well as the standardized the design of new spaces (EC 2.1);

2. all the old refrigeration systems have been censured to give life to a plan to replace the older chillers with new ones with energy class at least $\mathrm{A}++$ (EC 2.1);

3. the implementation of Smart Building Systems (BEMS) for HVAC plants and the adoption of an OpenData policy for energy consumption (EC 2.2);

4. the increase of renewable energy production, mainly due to three cogeneration plants, and to the adoption of a University policy in order to buy only renewable energy from the current Distribution System Operator (EC 2.3, 2.5).

Moreover, UniTo took several efforts to improve or to design elements of green buildings in a partecipatory way (EC 2.6), and there is an attempt to create sustainable design standards for university building designers.

\subsection{Community engagement}

Several initiatives have been organized during the past years in order to engage and involve both the academic community and external stakeholders in the rational use of energy.

Firstly, several round tables have been organized during the past two years in order to engage all type of stakeholders, from students to professors, from local non-governmental organizations (NGOs) to energy providers. A round table, titled UniToGO Community Engagement is organized each year for each working group - i.e. food, energy, waste, GPP and mobility - in order to collect direct feedbacks from UniTo's stakeholders on particular topics [15].

Secondly, seminars were organized on different arguments, such as smart metering systems, behavioural change, the Sustainable Development Goals, green roofs and crowdmapping, in order to explore and test new approaches, to understand psychological implications, opportunities, barriers and threats of new technologies and participatory processes. For instance, during a two days-event, titled "United for Sustainable UniTo", a workshop was run about "Internet of Thing for Energy Monitoring and Energy Efficiency" where students and participants could learn how to monitor temperature, humidity, energy consumption and other environmental measurements in real time - thanks to two open source projects: Scatol8 [16], based on Arduino, and OpenEnergyMonitor [17], based on Raspberry Pi - and how to build a basic circuits based on Arduino board [18].

In order to explore behavioural change, a workshop, titled "Co-Design of the local Sustainability Ecosystem" [19], was organized by engaging more than 40 local stakeholders from NGOs, public administrations, private enterprises, as well as citizens and students. The goal of the 4 hours workshop was to identify and represent the local ecosystem (i.e. 205 nodes and more than 300 links for the City of Turin), starting from four topics - energy, food, waste and mobility - highlighting the interrelations among them. In particular, related to energy topic, more than 60 relevant actors have been identified. Results from the workshop are publicly available [20] and are 
syncronized in real time with google drive spreadsheets [21]. As a final example, the "HOME" seminars, organized within the framework of a research project, "Human Observations Meta Environment" [22], based on a smart metering system for heating, ventilation and air conditioning (HVAC) systems, where buildings users may interact with buildings through natural interactions (gesture, voice and movements) or through social networks (Facebook, Instagram and Twitter) by writing feedbacks with a precise hashtag.

Above workshops and seminars, other type of actions and initiatives have been promoted in the past two years. For instance, "On Hot and Cold", an art installation, during the Contemporary Art Week in Turin, and "Hunt for $\mathrm{kWh}$ ", a flashmob to promote a rational use of energy where participants, split in teams, walk around the university buildings to switch off light, heating, desktop computers, printers and other electrical loads. "Hunt for $\mathrm{kWh}$ " was an initiative organized by the RUS network within the framework of "M'Illumino di Meno" (I enlighten me less) an annual Italian campaign [23] about energy saving. It is noteworthy also to mention the students' thesis on detailed energy audit which have helped the UniTo energy management in identifying many energy wastes [24-26].

\subsection{Research Projects}

Various innovative projets related to smart buildings and smart metering systems, as well as OpenData, have been lead within UniTo. A strategic research project, "ComfortSense" [27], started in 2014 and aiming to decouple human comfort from energy consumptions. ComfortSense is based on a smart metering system, which allows to measure objective variables (temperature, humidity, $\mathrm{CO}_{2}$ concentration and light intensity), and on a mobile app, which allows to measure subjective variables, i.e. direct feedbacks from buildings users. The project has been applied on three main buildings, engaging more than one thousand students and pointing out how it is possible to reduce energy consumption and, meanwhile, increasing human comfort.

Finally, an online dashboard for energy management has been developed [28], based on Open Source javascript library as D3.js [29] in order to help the analysis of energy consumption of a large buildings stock - as previously mentioned UniTo counts around 120 buildings - and to improve the efficiency of large energy audit. Further researches allow to develop and test multidimensional analysis tools in order to quickly recognize buildings depending on their main function and to identify and highlight outliers [30]. These analysis tools have been developed following the ASHRAE recommendations for a high quality and appropriate analysis method - i.e. accuracy, sensitivity, speed, reproducibility, ease of use, level of detail, availability of required data and quality of the output [31].

\section{Conclusions \& future planning}

\subsection{Education for sustainable development programmes}

The United Nations constituted 2005-2014 as the decade for educational sustainable development [32]. Since then universities start to debate about how they can form students capable of taking on the $21^{\text {st }}$ century challenges of global warming and climate change, social inequities, unsustainable lifestyles and the need to switch to renewable energy. UniTo started this process of greening curricula in 2015 with a brief research project on Green Jobs. Among others, one of the main project goal was to identify the green course already offered by the university, i.e. courses strictly related to environmental sustainability.

UniTo is one of the most ancient and prestigious Italian universities that virtually covers every field of knowledge (all disciplines, except for Engineering and Architecture) and offers a huge quantity of courses (around 8,000 different courses) every year. However, from the courses database it was not possible to simply identify the green ones. The project on green jobs run a qualitative analysis by interviewing several professors from different disciplines coming out with a result of 359 courses. Since then many green curricula have been developed in UniTo (Ph.D. Program in Innovation for the Circular Economy, Master's Degree Program in Economics of the Environment, Culture and Territory, Master's Degree Program in Socio Environmental Sustainability of Agrifood Networks).

Moreover, to answer the GreenMetric Education 6.2 indicator, UniTo developed a semiautomatic procedure to identify green courses by checking the occurrence of special keyword in the course and curricula descriptions. The testing of this procedure is still running comparing different sets of keywords.

\subsection{Environmental Sustainability Action Plan}

The last fundamental step towards the sustainability of the University of Turin has been achieved last October 10th, 2017, when the Academic Senate and the Board of Directors approved the "Environmental Sustainability Action Plan" (ESAP) [33], written in a collaborative and participatory way by UniToGO. The Action Plan was developed engaging dozens of UniTo stakeholders during the "UniToGO Community Engagement". During these round tables, internal and external stakeholders proposed possible actions and initiatives to improve the environmental sustainability of the University based on the three guidelines of UniToGO knowing, engaging, changing - and the five topics Food, Energy, Waste, Mobility and GPP. Within the ESAP of the University of Turin, dozens of actions, divided in structural, soft and communicative interventions, have been planned for the period 2017 2020. 


\section{References}

1. L., W. Petronella, Green curriculum: sustainable learning in higher education, The International Review of Research in Open and distributed learning 14, 1 (2013).

2. R., Pinheiro, P., V., Langa, and A., Pausits, One and two equals three? The third mission of higher education institutions, European Journal of Higher Education 5, 3 (2015).

3. International Sustainable Campus Network (ISCN): Available online at https://www.internationalsustainable-campus-network.org/

4. Unica-Green, Available online at http://www.unicanetwork.eu/page/unica-green

5. rootAbility gUG and the UNESCO Chair Higher Education for Sustainable Development at Leuphana University Lüneburg, Available online at http://www.greenofficemovement.org/

6. Commissione Sostenibilità Ambientale Del Senato Degli Studenti Dell'università Degli Studi Di Torino, Anno Academico 2007/2008, Available online at https://goo.gl/hXgEFP

7. Divisione Organizzazione e Finanza Staff Sistema di Programmazione e Controllo, Università degli studi di Torino Available online at https://www.unito.it/sites/default/files/fatti e numer $\mathrm{i}$ del piano di programmazione aa2009.pdf

8. The Green Public Procurement (GPP) Working Group, Available online at http://www.green.unito.it/en/Green Public Procure $\underline{\text { ment }}$

9. University of Turin, Available online at http://www.unito.it/ateneo/strutture-e-sedi/pianoenergetico-di-ateneo

10. The Energy Working Group, Available online at http://www.green.unito.it/en/Energy

11. University of Turin, Rapporto di sostenibilità 2015/2017, Available online at https://goo.gl/eDbPVV

12. Global Reporting Initiative (GRI), Available online at https://www.globalreporting.org

13. rootAbility, Available online at http://rootability.com/

14. RUS - Rete delle Università per lo Sviluppo sostenibile, Available online at https://sites.google.com/unive.it/rus/

15. UniToGO Community Engagement, Available online at http://www.green.unito.it/it/node/273
16. Scatol8, Available online at http://scatol8.net/

17. OpenEnergyMonitor, Available online at https://openenergymonitor.org/

18. GreenTo, Available online at http://www.unitixunitosostenibile.it/workshop/1/ind ex en.html

19. Workshop di Co-Design dell'ecosistema della sostenibilità, Available online at http://www.green.unito.it/it/Workshop CoDesign S ostenibilit $\% \mathrm{C} 3 \% \mathrm{~A} 0$

20. GreenTo, Available online at https://goo.gl/V83JDR

21. Stakeholder Sostenibilità Torino, Available online at https://goo.gl/QE6bVV

22. Human Observation Meta Environment, Available online at http://www.green.unito.it/it/home

23. Caterpillar, Available online at http://caterpillar.blog.rai.it/milluminodimeno

24. UniToGO, Sul Caldo e Sul Freddo, Available online at https://goo.gl/e1Es13

25. GreenTo, Innovation in the food sector From urban food production to the management of the university Available online at https://goo.gl/ns39QV -

26. UniToGO, L'Efficienza Energetica al Campus Luigi Einaudi: studio degli sprechi e idee per il risparmio, Available online at https://goo.gl/VPdHUU

27. ComfortSense, Available online at http://www.green.unito.it/en/ComfortSense Project

28. D., Cottafava, P., Gambino, M., Baricco, and A., Tartaglino, Energy efficiency in a large university: the Unito experience. Sustainable Built Environment. Towards Post Carbon Cities, 92-101 (2016)

29. M., Bostock, V., Ogievetsky, and J., Heer, D3: Data-Driven Documents. IEEE Transactions on visualization and computer graphics, 92-101 (2011).

30. D., Cottafava, P., Gambino, M., Baricco and A., Tartaglino, Multidimensional analysis tools for energy efficiency in large building stocks. 12th SDEWES conference (2017).

31. ASHRAE, Fundamentals American Society of Heating, Ventilating and Air- Conditioning Engineers (1997).

32. Shaping the Future, We Want - UN Decade of Education for Sustainable Development, UNESCO (2014) - Available online at https://goo.gl/q9PMmH

33. UniToGO, Approvato il Piano d'Azione per la Sostenibilità Ambientale, Available online at http://www.green.unito.it/it/ESAP_Unito 